\title{
Enabling IC Traceability via Blockchain Pegged to Embedded PUF
}

\author{
MD NAZMUL ISLAM and SANDIP KUNDU, University of Massachusetts Amherst, USA
}

Globalization of IC supply chain has increased the risk of counterfeit, tampered, and re-packaged chips in the market. Counterfeit electronics poses a security risk in safety critical applications like avionics, SCADA systems, and defense. It also affects the reputation of legitimate suppliers and causes financial losses. Hence, it becomes necessary to develop traceability solutions to ensure the integrity of supply chain, from the time of fabrication to the end of product-life, which allows a customer to verify the provenance of a device or a system. In this article, we present an IC traceability solution based on blockchain. A blockchain is a public immutable database that maintains a continuously growing list of data records secured from tampering and revision. Over the lifetime of an IC, all ownership transfer information is recorded and archived in a blockchain. This safe, verifiable method prevents any party from altering or challenging the legitimacy of the information being exchanged. However, a chain of sales record is not enough to ensure provenance of an IC. There is a need for clone-proof method for securely binding the identity of an IC to the blockchain information. In this article, we propose a method of IC supply chain traceability via blockchain pegged to embedded physically unclonable function (PUF). The blockchain provides ownership transfer record, while the PUF provides unique identification for an IC allowing it to be linked uniquely to a blockchain. Our proposed solution automates hardware and software protocols using blockchain-powered Smart Contract that allows supply chain participants to authenticate, track, trace, analyze, and provision chips throughout their entire life cycle.

\section{CCS Concepts: • Security and privacy $\rightarrow$ Hardware security implementation;}

Additional Key Words and Phrases: Blockchain, smart contract, traceability, supply chain, physically unclonable function, ownership transfer

ACM Reference format:

Md Nazmul Islam and Sandip Kundu. 2019. Enabling IC Traceability via Blockchain Pegged to Embedded PUF. ACM Trans. Des. Autom. Electron. Syst. 24, 3, Article 36 (April 2019), 23 pages.

https://doi.org/10.1145/3315669

\section{INTRODUCTION}

The globalization of semiconductor manufacturing has increased the risk of tampered and counterfeit products from manufacturing to distribution and field use. Malicious actors can counterfeit, tamper with, or re-package ICs and introduce compromised ICs into a supply chain [27, 34]. This exacts financial loss to legitimate suppliers [61] and poses a security risk for safety critical applications, like defense [35,59]. In an effort to stem the tide of counterfeit electronics, traceability has long been established and promoted [55]. Traceability plays a key role to secure the supply chain

Authors' addresses: Md N. Islam and S. Kundu, Department of Electrical and Computer Engineering, University of Massachusetts Amherst, Amherst, MA, 01003, USA; emails: \{mislam, kundu\}@umass.edu.

Permission to make digital or hard copies of all or part of this work for personal or classroom use is granted without fee provided that copies are not made or distributed for profit or commercial advantage and that copies bear this notice and the full citation on the first page. Copyrights for components of this work owned by others than ACM must be honored. Abstracting with credit is permitted. To copy otherwise, or republish, to post on servers or to redistribute to lists, requires prior specific permission and/or a fee. Request permissions from permissions@acm.org.

(C) 2019 Association for Computing Machinery.

1084-4309/2019/04-ART36 \$15.00

https://doi.org/10.1145/3315669 
by counterfeit electronic parts avoidance, detection, mitigation, and disposition. Hence, it is imperative to develop a secure IC traceability system that can allow both the supplier and a potential customer to verify its provenance.

Traceability refers to the combination of the ability to know the current possession of a product at all times (track) and the ability to find the origin, ownership history, time spent at each point (trace), by means of recorded identifications [40,42]. Tracing allows a customer to establish a product's provenance with a high degree of confidence. Tracking can help suppliers minimize counterfeit products as well as offer value-added services, like product recall during the lifecycle of a product.

Prior works proposed implementation of a centralized system with a governing third party to empower supply chain traceability to ensure data and transaction transparency over a product's lifetime. The governing third party is commissioned with the task of creating a centralized data storage to enable a flow of trusted information [31, 58]. However, relying on a governing third party to broker all data about every product's supply chain may create a single point of weakness and an inherent bias, fraud in the system. If the party were the brand itself, or the most powerful actor in the supply chain, then it would be responsible ultimately for only its own bottom line; this could lead to selective disclosure or, worse, extortion. If the supply chain data were gathered by a third party, then it would have to be both totally unbiased and properly incentivized to deliver the technical capability of running the system. Third parties like NGOs or industry associations, however, would become a single point of weakness; this would make them and their operations a vulnerable target for bribery, social engineering, or targeted hacking. For example, GS1 is a standards organization that tries to solve supply chain problems by uniquely identifying and accurately capturing information about products. However, according to recent Data Quality pilots, data accuracy was a growing problem-about $50 \%$ of the data surveyed was inaccurate [2]. Besides, GS1 have, of late, been restricting access to centralized product database, excluding consumers and prioritizing corporations [3]. Moreover, although few supply chains are integrated, their supporting information systems can be heavily fragmented [29]. Hence, we need to come up with a more robust approach for developing IC traceability. Potential solutions must also account for legitimate re-sale of devices during the product lifetime.

In this article, we propose a novel IC traceability protocol via blockchain-based ownership management system. A blockchain is an immutable database that maintains a continuously growing list of transaction records secured from tampering and revision [88]. Each block contains a list of transactions, timestamp, nonce, and a link to the previous block, forming a chronological chain. Often introduced as the technology behind Bitcoin [57, 75], blockchain has the potential to revolutionize numerous industries beyond financial services [7, 36, 80, 82]. It can enable the IC ownership transfer information to be verified, recorded, and prevent any malicious party from altering or challenging the legitimacy of the information recorded. Thus, this verifiable and immutable ledger can enable identification and traceability of an IC throughout the supply chain and its deployment lifetime.

However, including just the ownership or basic IC information in the blockchain is not sufficient enough. To corroborate a record in the blockchain against a physical device, the device must be authenticated securely and uniquely. For this purpose, we utilize physical unclonable functions (PUFs) as the hardware root-of-trust. PUFs are innovative, lightweight circuit primitives that harness the intrinsic disorder in an IC introduced during the fabrication process. Various PUFs have been proposed and developed in recent years and are generally classified as either Strong or Weak PUFs. Strong PUFs $[13,84]$ can enable secure, low-cost authentication using Challenge-Response Pairs (CRP) [79]. The start-up behavior of a Weak PUF [32] can also be used for unique identification and authentication $[5,25]$. In this article, we propose a method of securely and uniquely 
identifying an IC using both Strong and Weak PUF to corroborate the blockchain information. The primary focus of this article is specifically on securing IC supply chain, although the methodology can be applied to any electronic device supply chain.

For automated execution of enrollment, authentication, ownership traceability, and ownership transfer of an IC, we deploy smart contract in blockchain. Smart contracts are blockchainpowered automated computer programs that, once started, automatically execute the conditions pre-programmed $[10,81]$. The main advantage of deploying smart contracts in a blockchain is the assurance provided by the blockchain that the contract terms cannot be modified. In our proposed smart contract, the contractual clauses regarding enrollment, authentication, and ownership transfer of an IC is translated into embedded hardware and software. The embedded hardware and software can self-verify that conditions have been met to execute the contract.

The major contributions of this article are:

- Proposing a blockchain-based open traceability protocol that IC suppliers can use to track supply chain.

- Enhancing the traceability protocol, such that, customers can trace and verify IC's provenance.

- Proposing a smart contract that automates self-execution of the enrollment, authentication, and ownership transfer of an IC.

- Developing a comprehensive ownership management system that enables each party along the supply chain to prove the ownership of PUF-embedded IC and transfer it to a new owner.

- Demonstrating the proposed solution in Ethereum blockchain. The code has been made publicly accessible in Github [1].

The key novelty of this work is developing blockchain-based traceability protocol for ensuring that the IC supply chain can be fully tracked and traced from the time of fabrication to the end of product-life. Several works have been done to deploy blockchain across a wide span of industries: from finance [46] and health-care [45], to utilities [50], real estate [56], government sector [86], and IoT $[21,33]$. To the best of our knowledge, no other authors have used blockchain for IC traceability.

The article is organized as follows: Section 2 describes the related works on IC supply chain traceability. Section 3 briefly presents backgrounds related to our proposed methodology for establishing IC supply chain traceability using blockchain. Section 4 introduces the system requirements and implemented smart contracts for our proposed protocol. In Section 5, we present our proposed methodology to establish IC supply chain traceability using blockchain and embedded PUF. Section 6 details about the authentication method used in our traceability protocol. Section 7 discusses about how the proposed protocol performs the counterfeit avoidance and detection. Section 8 discusses security, privacy and performance analysis of the proposed protocol. Section 9 outlines a demonstration of the protocol and discusses future directions. Finally, Section 10 concludes the article.

\section{RELATED WORKS}

Several traceability solutions have been proposed in the literature for counterfeit detection in IC supply chain. These solutions resort to RFIDs, various kinds of chip IDs, package IDs, blockchain, and so on. In this section, we present the literature works that proposed various IC traceability solutions.

RFID-based Traceability: RFID-based counterfeit detection has been proposed in References [22, $74,78,91]$. Schuster et al. propsed an RFID-based track and trace solution using EPC (Electronic 
Product Code) Network infrastructure [48]. Staake et al. proposed an extension of EPC Network infrastructure with an EPC Product Authentication Service to provide secure authentication functionalities [78]. Shi et al. proposed a Batch Clone Detection (BCD) scheme that performs the clone tag detection at a batch level [74]. The proposed scheme reduces storage and computational overheads on the centralized detection server side. Elkhiyaoui et al. proposed a new protocol, CHECKER for counterfeit detection in RFID-based supply chains through on-site checking [22]. In this protocol, RFID readers can verify product genuineness by checking the validity of the product's path, while RFID-equipped products travel through the supply chain. However, RFID-based technologies cannot provide truly secure solutions for supply chain traceability, because an adversary can easily copy the unique identifier of one RFID tag to another tag.

Package ID-based Traceability: Several traceability solutions proposed affixing a unique ID in package (SHIELD [15]), on package (DNA marking [55], nanorods [49], QR codes) of each component to track it, as it moves throughout the supply chain. For verifying the trustworthiness of an electronic component, DARPA initiated a program called SHIELD (Supply Chain Hardware Integrity for Electronics Defense) [15]. The program proposes to embed a dielet into host packages of legitimately produced ICs, without disrupting or harming the system. A secure remote server stores the information for identification and authentication, such as cryptographic key and serial ID for each dielet. Passive sensors inside the dielet can record any malicious behavior as tampering evidence. Jin et al. proposed an improved protocol that resists the try-and-check attack in DARPA's example authentication protocol [43]. The proposed two-phase activation secures the untrusted transit between a trusted fabrication facility and assembly facility. A true random number generator (TRNG) inside the dielets efficiently generates their cryptographic keys and serial IDs in parallel during a trusted fabrication process.

Chip ID-based Traceability: Several works have proposed traceability solutions using various kinds of chip IDs, such as, PUFs, ECIDs [67], and so on. Skudlarek et al. proposed a hardware rootof-trust-based solution that enables connection of SoCs to a secure server [76] for tracking them at each step in the supply chain. An SRAM PUF provides each chip with its unique ID and DNA. The chip connects to a secure server at important stages in the supply chain. The server authenticates the chip and records that event, establishing a reliable audit trail for the chip's progress and providing proof of provenance. Several Strong PUF-based RFID ICs have also been proposed previously for supply chain traceability $[18,65,83]$. In these methods, the back-end server stores a list of challenge-response pairs for RFID tags with embedded PUF. When the RFID tag communicates with a reader, the back-end server sends a challenge and the tag's PUF reconstructs and transmits the corresponding response. If the response matches with the one stored by the back-end server, then the tag is verified. In all these methods, RFID monitoring schemes need to have either a persistent online connection between supply chain partners and the back-end database or a local database on each partner site to adequately perform authentication. However, this approach is not secure from man-in-the-middle attack. ECID-based chip tracking solutions [67, 90] are not secure. As it is static and readable, it is easy to clone an ECID. Moreover, the chip vendors are silos. Individual companies do not share data across corporate boundaries and cannot build cross-party trust relationships.

Blockchain-based Traceability: In Reference [37], the authors propose a blockchain solution for IC traceability, where customized transactions are used for logging supply chain information in blockchain. However, the proposed solution does not detail a method for transaction automation in an already existing blockchain platform. In Reference [26], a method is proposed to establish IoT authentication using PUFs in combination with blockchain. The proposed solution targets securing the integrity of IoT data with PUF derived keys and uses blockchain for storage, 
transparency, auditability of the IoT data. The proposed solution explores its benefits to multifactor authentication, software integrity and continuous authentication of IoT devices. However, it does not facilitate traceability solutions and ownership management to ensure the integrity of supply chain, from the time of fabrication to the end of product-life, which can allow a customer to verify the provenance of a device or a system.

Hence, a more comprehensive protocol is required to enable IC traceability, authenticate a device, and transfer the ownership for a secure supply chain. In this article, we introduce a novel method for IC traceability via blockchain-based smart contract. A blockchain-based traceability solution can increase transparency, add end-to-end visibility, maintain a common single version of the truth about supply chain, and provide cyber-attack resilience for data storage via no single point of failure. Blockchain establishes a trusted environment for all participants-manufacturers, distributors, retailers, and consumers, who can gain permissioned access to known and trusted information regarding the origin and state of their transactions.

\section{BACKGROUND}

In this section, we introduce some relevant concepts related to our blockchain-based IC traceability protocol. First, we discuss about blockchains required for immutable IC ownership record and smart contract required for automated execution of enrollment, authentication, ownership traceability, and ownership transfer of an IC. Next, we discuss about PUFs that are used for securely and uniquely authenticating the IC to corroborate the blockchain information.

\subsection{Blockchain}

Blockchain is a distributed ledger that maintains a continuously-growing list of ordered records called blocks. Each block contains a list of transactions, timestamp, nonce, and a link to the previous block, forming a chronological chain. A transaction is a transfer of any asset with value from the current owner to a new owner that is broadcast to the blockchain network and after being validated, collected into blocks. Each block is reviewed by network participants or nodes before it is added to the chain. When the nodes agree via a consensus that the information within a block is correct, then the block is appended to the blockchain without the need of central authority. Figure 1 illustrates a blockchain formed from a sequence of blocks, each containing multiple transactions. The distributed ledger facilitates efficient business networks wherever anything of value needs to be tracked and traded without requiring a central purpose of management. By design, blockchains are inherently immune to modification of the data once recorded. Users can view it and add to it, but cannot change the information that is already there.

3.1.1 Blockchain Classification. There are three types of blockchain: public, private, and consortium. Public blockchains are accessible to every Internet user. The public nature stems from the fact that everyone in the blockchain network can freely and unconditionally participate in the consensus process-the process of determining what blocks are added to the chain, and what its current state is Reference [9]. A private blockchain is a blockchain where write permissions are kept centralized to one organization.

In a consortium blockchain, a pre-selected group of organizations control the consensus process instead of allowing any user within a blockchain network to participate in the consensus process or allowing a single organization to have full control. The right to read the blockchain can be made public, or restricted to the participants in a consortium blockchain. A hybrid approach is also possible that allow members of the public to make a limited number of queries [9].

3.1.2 Smart Contract. Smart contracts are blockchain-powered autonomous computer programs that, once started, execute automatically the conditions defined beforehand, such as the 


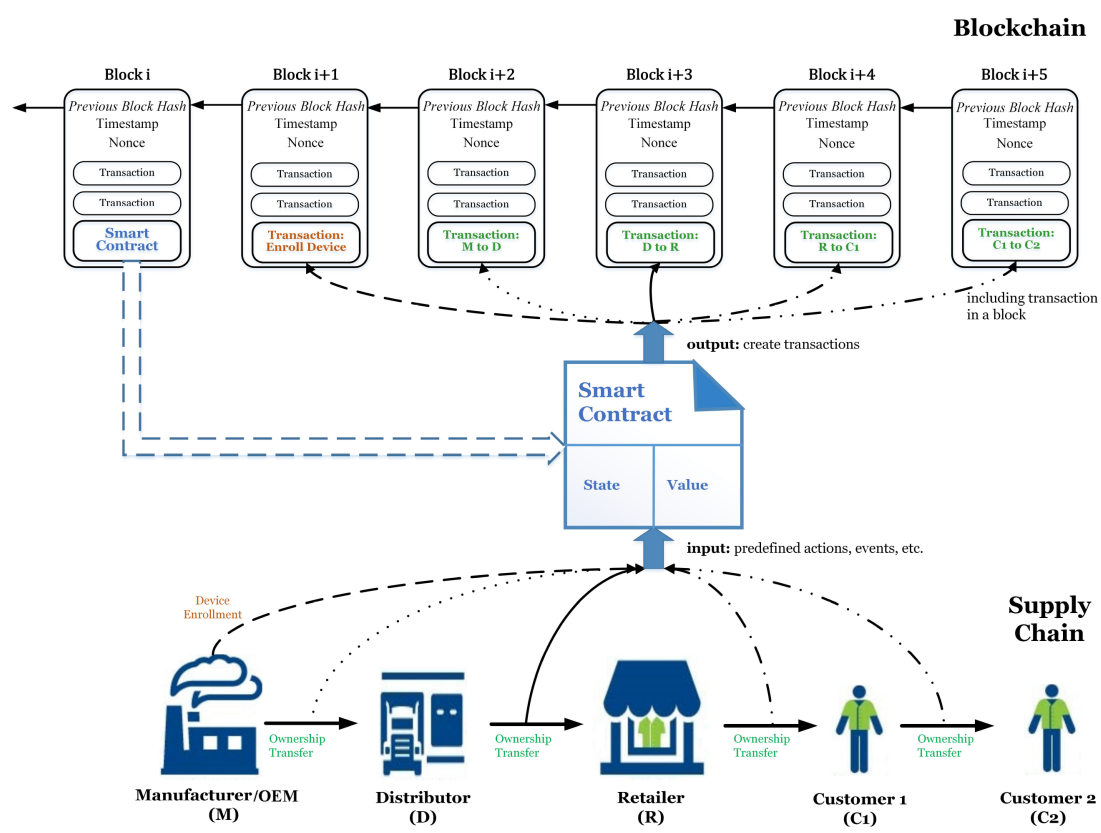

Fig. 1. Proposed approach for IC traceability from manufacturer to the end-user. OEM: Original Equipment Manufacturer.

verification, facilitation, or enforcement of the negotiation or performance of a contract [81]. Smart contracts give us distributed trustworthy computations on blockchain platform. They translate the existing contractual clauses into embedded hardware and software in such a way that it can selfverify that conditions have been met to execute the contract [10]. Smart contracts contain code functions and can interact with other contracts, make decisions, store data, and send tokens/money to others.

The main advantage of deploying smart contracts in a blockchain is the assurance provided by the blockchain that the contract terms cannot be modified. The blockchain makes it impossible to modify or tamper the contract terms. It generates the confidence and security necessary to automate the declarative phrases without resorting to a third party.

\subsection{PUFs}

Physical unclonable functions (PUFs) harness the intrinsic disorder in an IC introduced during the fabrication process and provide a set of unique input to output mappings, called challengeresponse pairs (CRPs) [18, 79]. Based on the implementation, a PUF that provides a limited set of CRPs is classified as a Weak PUF, while a design that can produce an exponential number of CRPs is called a Strong PUF.

3.2.1 Strong PUFs. Strong PUFs can be leveraged for an authentication mechanism to uniquely identify an IC and detect tampering, impersonation or substitution of such components. The salient features of a Strong PUF are uniqueness of responses across different PUFs to the same challenge; ensuring reliability of a response in the presence of noise; and unclonability in the form of being resistant to model-building attacks [70]. Even if a Strong PUF exhibits ideal uniqueness and unclonability [85], it can still be susceptible to noise. Hence, authentication using a Strong PUF requires acquiring multiple CRPs to check against a database and applying a threshold to account 
for noise. The number of CRPs required depends on the total population of possible devices that need to be distinguished and the amount of noise that may affect the system [64].

3.2.2 Weak PUFs. Weak PUFs can be leveraged for secure cryptographic key generation to combat semiconductor device counterfeiting, theft of service and tampering. Weak PUFs rely on intrinsic process variations to produce repeatable and unique fingerprint. This fingerprint is further processed to generate a unique cryptographic key. For generating a reliable key, the fingerprint needs to be reproducible over time, even under changing environmental conditions. However, noise in the system can affect the fingerprint and introduce errors. To alleviate noise and generate stable keys, a number of solutions have been proposed, such as, fuzzy extraction and error correcting codes [17], accelerated device aging [38, 39], built-in self-test [8], and so on.

\section{SYSTEM REQUIREMENTS AND SMART CONTRACT IMPLEMENTATION}

In this section, we outline the key system requirements and implemented smart contracts for our proposed IC traceability protocol. First, we describe the approach and operational requirements of our protocol. Next, we explain the implementation of smart contract for IC traceability.

\subsection{Approach}

The blockchain will contain the record of relevant IC ownership transfer information, termed as a transaction, and PUF data, used for authentication, at each point of supply chain over the device's lifetime. This enables IC traceability and the proof-of-ownership without an explicit need for trusted intermediary. Furthermore, authorized parties can utilize the PUF data in blockchain to authenticate, track, analyze, and provision chips. Figure 1 presents our proposed approach using blockchain.

Before detailing the protocol, we enumerate the key requirements for creating the blockchain and explain their necessity for enabling reliable IC traceability.

(1) Only the legitimate IP owners registered with a designated consortium can claim the initial ownership of an IC and write the relevant PUF data in the blockchain.

(2) Only the current owner of the IC is able to create a new transaction for transferring the ownership to a new owner.

The first requirement prevents unauthorized parties, like counterfeiters, from falsely claiming ownership of an IC. This can be ensured by verifying that the party enrolling a device is registered with a consortium. The consortium can be formed of multiple semiconductor organizations, each of which operates a node in blockchain. Examples of such group of organizations can be Semiconductor Industry Association (SIA), Joint Electron Device Engineering Council (JEDEC), and so on. Validation of a transaction requires a set number of nodes to sign-off on it.

The second requirement prevents an unauthorized party from hijacking the ownership of an IC by creating a fraudulent transaction. Our protocol grants only the current owner the ability to create a new transaction. The current owner's identity is established using the information in the last transaction recorded in the blockchain and also, by utilizing PUF authentication to verify physical possession of the IC.

Both of the above mentioned key system requirements for IC traceability can be fulfilled by implementing a smart contract in the blockchain. In the next section, we detail the smart contract implemented in our IC traceability protocol.

\subsection{Implementation of Smart Contracts}

In our proposed protocol, a smart contract defines the condition for enrolling a new device by a manufacturer and transferring a device to a buyer by the current owner. It also defines the 
function for checking the ownership and authenticating the device. In this section, we describe all the elements needed for implementing a smart contract.

4.2.1 Ownership Keys and Addresses. All the supply chain participants are registered with consortium blockchain and assigned private-public key pairs. This is necessary for uniquely identifying a supply chain participant and preventing any forgery. First, a 256-bit private key is generated from which the public key is derived. The private key must be kept secret. Each transaction is supplemented with a digital signature created using the private key. The signature uniquely identifies any supply chain participant,

$$
K_{\text {priv }} \in\{0,1\}^{256} \text {. }
$$

Next, a 512-bit public key is generated from the private key using ECDSA (Elliptical Curve Digital Signing Algorithm) algorithm. The major advantage of ECDSA over other Public Key Cryptography (PKC), such as RSA is that ECDSA uses much smaller keys and signatures to achieve the comparable level of security [44]. The public key is used for verification of the transaction signature. In ECDSA protocol,

$$
K_{\text {pub }}=E C D S A_{512}\left(K_{\text {priv }}\right) .
$$

An owner embeds the public key in any transaction and then signs the transaction with his or her private-key. Since the 512-bit public key is large, it is converted to a smaller address that can be shared with others and utilized as part of the blockchain transaction. To prevent length extension attack [24], we adopt a double hash scheme [14], where the 512-bit public key is hashed using SHA-256, which is further hashed using RIPEMD-160 to generate the 160-bit address,

$$
\mathrm{K}_{\text {address }}=\operatorname{RIPEMD}_{160}\left(\mathrm{SHA}_{256}\left(\mathrm{~K}_{\text {pub }}\right)\right) \text {. }
$$

The consortium plays a key role in authenticating participants and it maintains a directory that binds the identity information of an associated party with transaction address. Any blockchainbased identity management system can ensure the reliability of user's entity information [6, 53, 66]. A user can manage personal keys and addresses using a digital wallet, which can be used to perform any transaction. A digital wallet is software and hardware, or specifically designed hardware, that holds the private-public keys and the address [23].

4.2.2 Smart Contract Implementation by the Consortium. To enable IC traceability, we have implemented smart contract, namely, ownershipContract (OC). The contract is created by the consortium to maintain uniform applicability and usability for all supply chain participants. After creating the smart contract, it is sent to the blockchain network as a transaction that assigns an address to the contract. After this initial transaction, the contract becomes forever a part of the blockchain and its address never changes. Smart contract, OC provides the services for device registration, checking the ownership information, device authentication and ownership transfer. Next, we explain these services and how these are implemented by our proposed smart contract.

Device Registration: For introducing a device into the supply chain, the device must be registered first. This is implemented by the smart contract function registerDevice(). Algorithm 1 presents the pseudo-code of function registerDevice(). The function enrolls a device if the message sender is one of the manufacturers registered with the consortium. The deviceInfo in the function includes data for identification and authentication. The identification data can be a serial number fo the device (e.g., Electronic Product code (EPC)). It is used to look up the targeted device being queried among a collection of devices. Here, the device identifier is being used for the purpose to identify, and not as the primary means to authenticate. For authentication purpose, manufacturer includes PUF data as input to the function registerDevice(). 


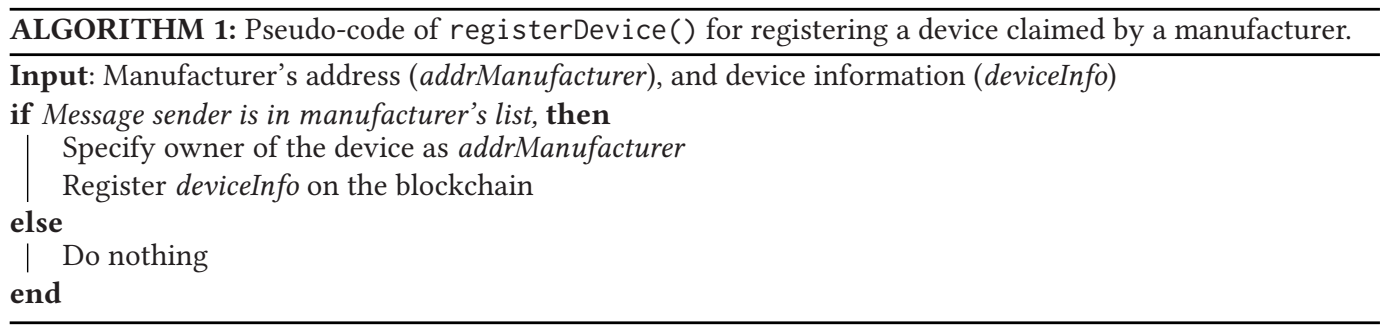

Ownership Verification: Before buying a device, a potential buyer needs to verify the ownership to confirm that the owner is a genuine one. This is implemented by the smart contract function checkOwnership(). Algorithm 2 presents the pseudo-code of function checkOwnership(). This function verifies the ownership of the device against the seller's address. If the device with the provided identifier is owned by the seller, then it returns True. The function is invoked when any potential buyer wants to verify the ownership of the device. Additionally, the function can return the ownership history till the provenance.
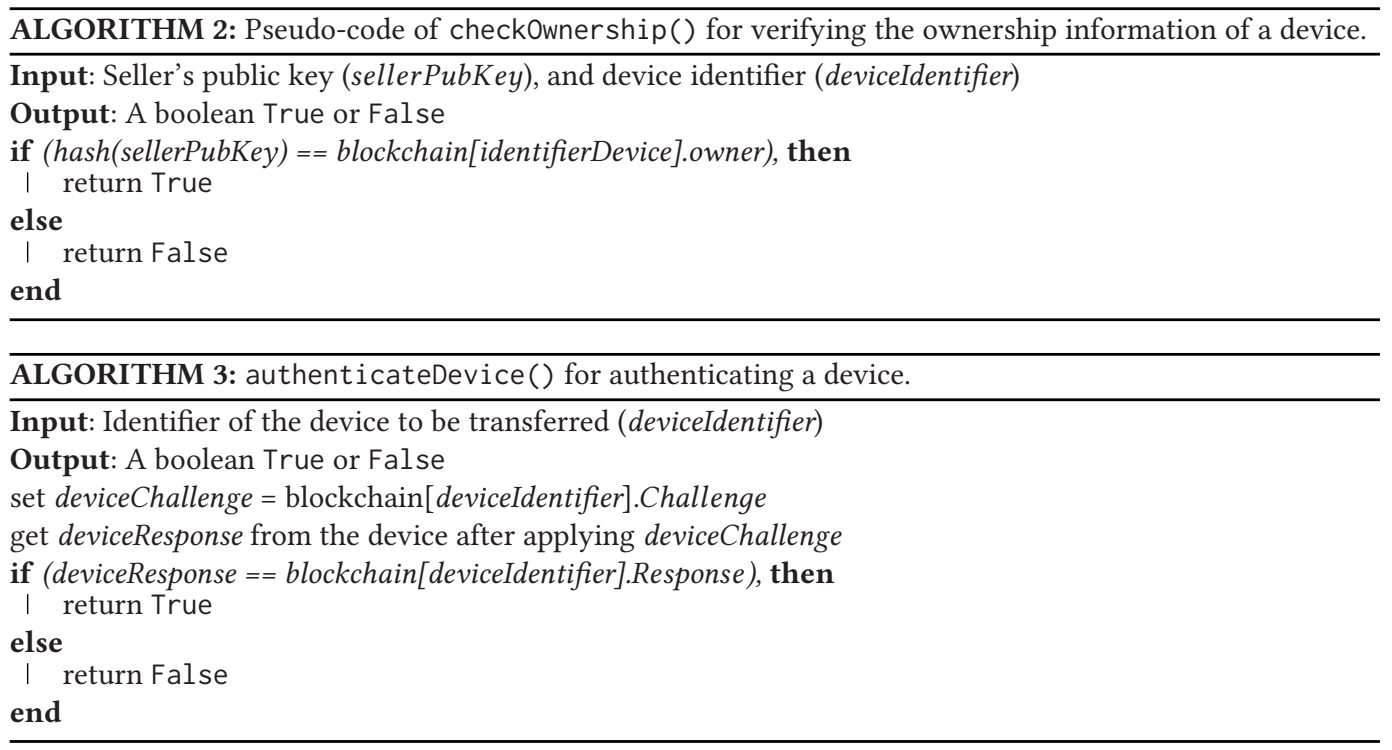

Device Authentication: A potential buyer must be able to authenticate a device before buying to confirm that the device is authentic; not a counterfeit one. This is implemented by smart contract function authenticateDevice(). Algorithm 3 presents the pseudo-code of authenticateDevice(). This function starts device authentication process for a given device identifier. The process includes getting the challenge-response data for particular deviceIdentifier, applying the challenge to the device via verifier's wallet, calculates the response and matching the challenge-response pairs.

Ownership Transfer: For transferring the ownership of a device, the smart contract implements the function transferOwnership(). Algorithm 4 presents the pseudo-code of transferOwnership(). This function transfers the device (deviceIdentifier) from the seller (addrSeller) to buyer (addrBuyer). First, the function checks whether the message sender is the owner of the device with deviceIdentifier. If that is true, then the function assigns the addrBuyer as the new owner of the device. 


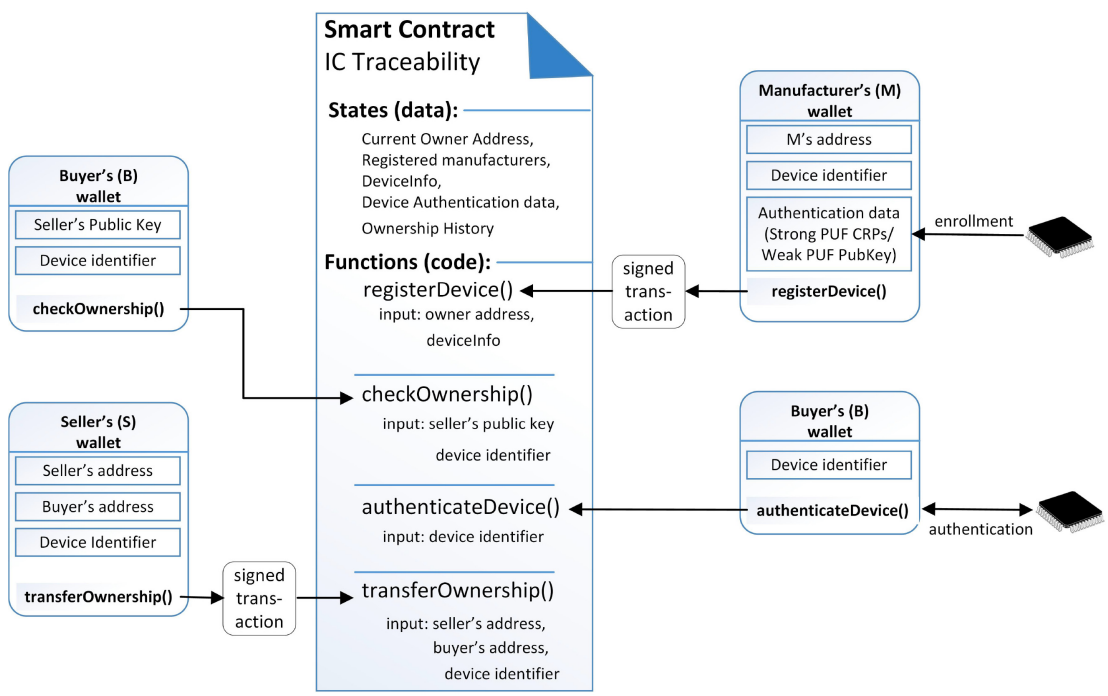

Fig. 2. Smart contract implementation for IC traceability.

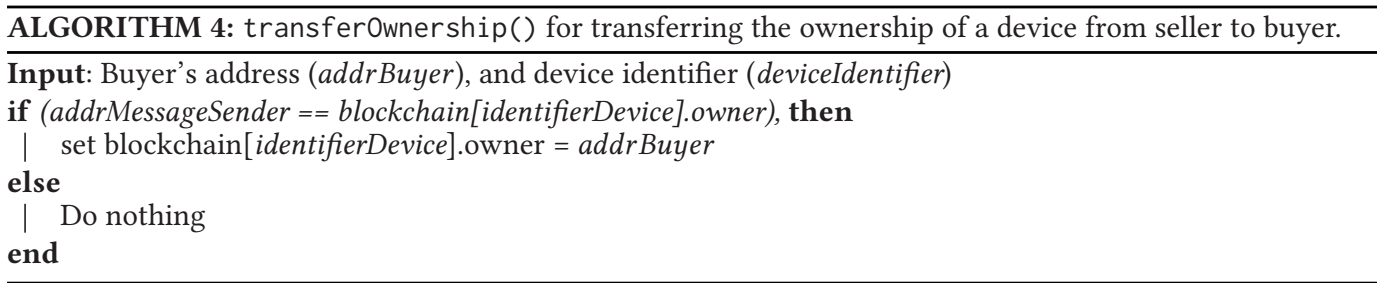

\section{PROPOSED IC TRACEABILITY PROTOCOL}

In this section, we present the details of the algorithmic procedures between all parties necessary for the realization of the proposed protocol. The procedure for enrolling a device by the manufacturer is first outlined. This is followed by the procedure for transferring the ownership, which is the key part of our overall traceability protocol.

\subsection{Enrollment of a Device by the Manufacturer}

When a manufacturer (M) wants to register a device, it sends a transaction registerDevice() to the smart contract OC (Figure 2). The manufacturer also sends necessary device information, such as, identifier of the device, authentication information (PUF data) in the transaction. We name the first transaction for the enrollment of a device as genesis transaction. The manufacturer can also enroll $\mathrm{N}$ number of devices in the same transaction by including corresponding information of all the devices. This facilitates scalability of the protocol. As all the blockchain transactions are digitally signed by the transactor, any counterfeiter cannot illegally claim to be a non-authorized manufacturer.

\subsection{Procedure for Ownership Transfer}

Figure 3 illustrates the detailed system model of the proposed ownership transfer protocol. In this work, we denote the current and the new owners as the seller and buyer, respectively. The protocol consists of several phases described as follows: 


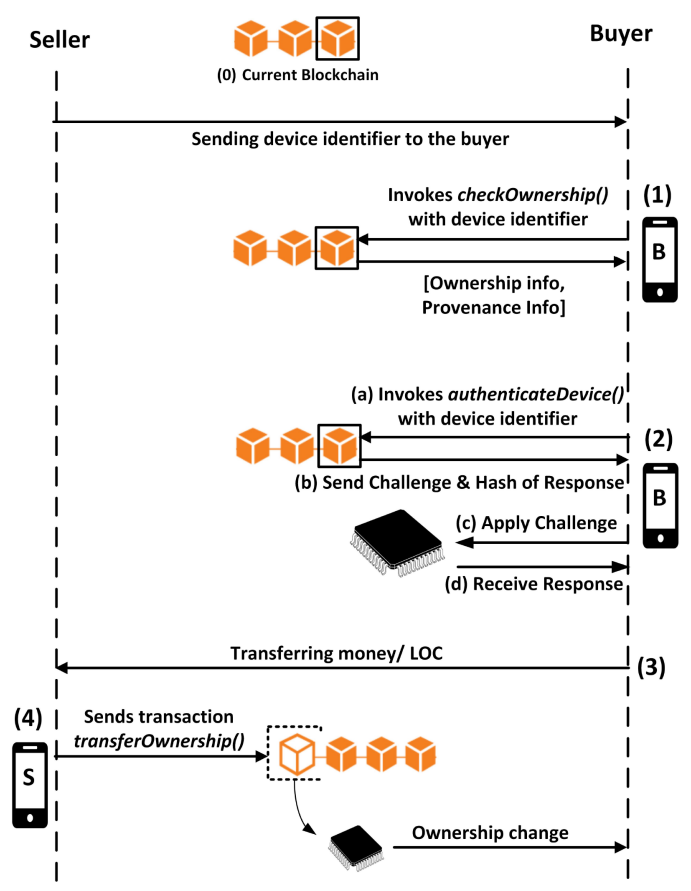

Fig. 3. Detailed diagram of the ownership transfer protocol.

5.2.1 Verifying the Ownership Information. To verify the current ownership information, the buyer invokes function checkOwnership() of the smart contract. If the return is True, then the buyer can verify the genuineness of the current seller. Buyer can also trace back all the way along supply chain to IC's provenance.

5.2.2 Authenticating the IC. Verifying the ownership of a device is not sufficient enough. A malicious seller can replace the original device with a counterfeit one and sell it to the buyer. Therefore, the buyer also needs to authenticate the device with the stored blockchain information. We propose two implementations for authentication of the device. The details of the implementation are presented in Section 6.

5.2.3 Making Payment. Once the IC is authenticated and the seller's IC ownership is verified, the buyer makes a payment to the seller. The payment can be made in terms of Letter of Credit (LOC) and only after the seller completes a transaction later, the money is transferred. This ensures that if a seller does not write the transaction, he will not get payment. Additionally, all the terms of the contract, trade documentation, and LOC can be recorded by blockchain-based smart contract for further safety of any ownership transfer payment [16, 72]. However, details of secure payment is out of our focus in this article.

5.2.4 Implementing Transaction for Ownership Transfer. In the last step of the proposed protocol, the seller issues a transaction transferOwnership() with the device identifier and the buyer's address. This function transfers the device with (deviceIdentifier) from the seller to the buyer.

\section{AUTHENTICATION BY STRONG AND WEAK PUFS}

During ownership transfer, the buyer needs to authenticate an IC with the stored blockchain information. We propose two implementations for authenticating the device. In this section, we present the details of the implementations. 


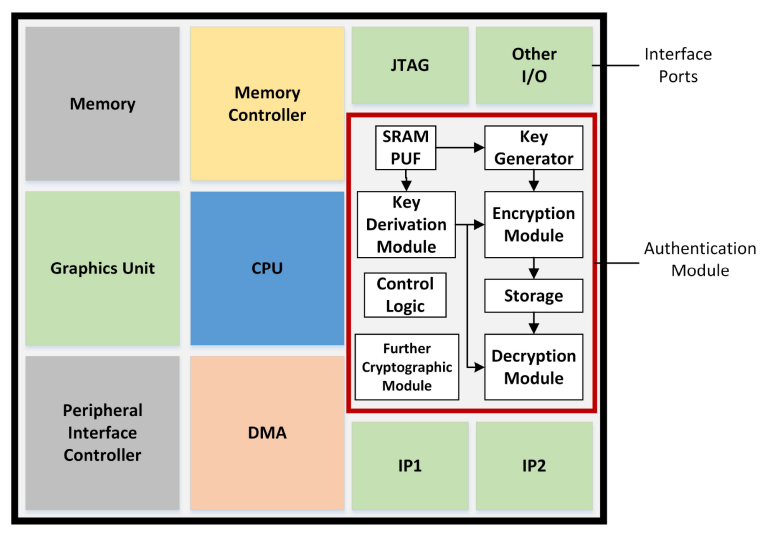

Fig. 4. Hardware authentication module for generating cryptographic key from PUF embedded in an SoC [28].

\subsection{Authentication via Strong PUF}

When a manufacturer registers a device, it sends a transaction registerDevice() to the smart contract OC. This transaction includes necessary device information, such as, identifier of the device and Strong PUF CRPs for future authentication. At any point of supply chain over the device's lifetime, a potential buyer can authenticate a device by invoking autheticateDevice() with the respective deviceIdentifier. This retrieves challenges from the blockchain and the challenges are applied to the IC. The PUF embedded in the IC will give the corresponding responses. If the calculated hash of the responses matches with the hash recorded in the blockchain, then the IC is authenticated. Hash is a compact representation of the entire digital content, which is easy to compute and verify. For example, while pushing software updates, software vendors supply a hash (also known as digest), which is digitally signed by the software vendor. This allows the devices to verify authenticity of the software update by computing hash and verifying it using vendors public key. Inspired by this software attestation technique, we propose compressing the CRP table as digest, which can similarly attest the authenticity of PUF CRPs supplied by the manufacturer.

For achieving pervasive authentication, it is necessary that the authentication functionality be easily integrated with modern electronic devices, such as smartphones and also, be easy to use. One such example, as illustrated in Figure 3, shows an IC verification process using Near Field Communication (NFC). Several PUF NFC tags are already used in commercial products [92]. For an easy authentication process, a silicon PUF can be integrated into a form of NFC. With the recent emergence of NFC-enabled smartphones, custom wired authentication system setup is no longer necessary for many use cases. This facilitates anyone in the supply chain to perform authentication of ICs by simply using a smartphone with the necessary application. Other possible authentication procedures can also be implemented based on the needs of the system.

Here, we note that if the distributors and retailers have no authenticating infrastructure at all, they can still lookup the blockchain using device identifiers and verify the provenance of the chips. However, in this case, they cannot authenticate the chips. An end customer can always verify the provenance and authenticate a chip.

\subsection{Authentication via Weak PUF}

Several Weak PUF-based public key cryptography for authentication have been proposed in the literature $[5,8,25,52]$. Figure 4 presents a hardware authentication module to generate 


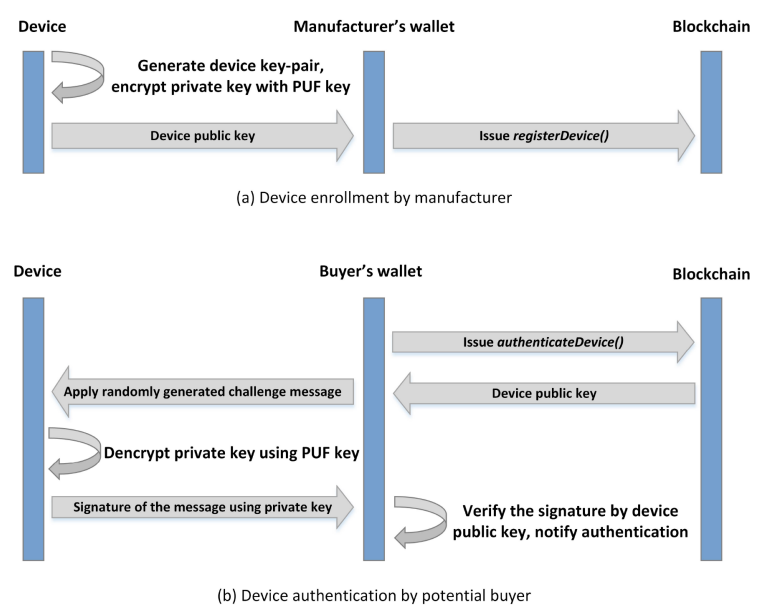

Fig. 5. (a) Device enrollment and (b) authentication process via Weak PUFs.

cryptographic key from SRAM-based Weak PUF [28]. The manufacturers first records Weak PUF data in the blockchain by issuing a transaction registerDevice(). At any stage of the supply chain, any buyer can authenticate the device by invoking authenticateDevice(). The components of the authentication module and their functions are described as follows:

6.2.1 Key Generation Module. During enrollment phase, the key generator in the authentication module generates a private-public key pair. Using the private key, a device can create signature of a message protecting the message's integrity and proving its authenticity. At a receiving end of message, the digital signature can be verified for authenticity using the public key corresponding to the private key. The private-public key pair may be keys for RSA, DSA, Schnorr, El Gamal, Elliptic Curve-based public key cryptosystems, and so on. For the purpose of illustration, we describe RSA public key cryptography here. First, the key generator finds two prime numbers from a seed, which is derived from PUF output, e.g., the contents of an SRAM. Finding prime numbers can be done on an appropriately programmed device or Hardware Security Module (HSM). Once two prime numbers of appropriate sizes are found, an RSA private-public key pair is constructed. The RSA key pair generation is computationally intensive process and done only during enrollment phase.

6.2.2 Encryption Module. The private key is encrypted with a second key (encrypting key) generated from the same PUF and stored in a non-volatile memory. This encryption is done using symmetric encryption algorithm, like AES and the encrypting key is obtained from any random 128-bit string from the PUF. The manufacturer records the public key in the blockchain by issuing a transaction registerDevice() for the device (Figure 5(a)).

6.2.3 Decryption Module. During authentication phase, the encrypting key can be generated instantly from the PUF output. Using the encrypting key, the decryption module generates the private key of the device.

When a potential buyer wants to authenticate the device, he invokes authenticateDevice() with the device identifier. The smart contract sends the device's public key to buyer's wallet. The buyer's wallet then sends a challenge message generated with a pseudo-random number generator to the device. The device's further cryptographic module creates the device signature using 
the private key. Using the public key, the buyer's wallet can verify the digital signature for the authenticity of the device as shown in Figure 5(b).

\section{COUNTERFEIT DETECTION BY THE PROPOSED TRACEABILITY PROTOCOL}

Various ways of counterfeiting ICs have been described in the literature [27]. In this section, we present how our proposed traceability protocol prevents specific counterfeit mechanisms.

\subsection{Recycled \& Remarked ICs}

Recycled and remarked components jointly contribute more than $80 \%$ of counterfeit incidents [47]. The recycled ICs are taken from used printed circuit boards (PCBs), repackaged and remarked, and then sold in the market as new. Our proposed protocol defeats this malicious approach in the following two ways:

If an unregistered, malicious manufacturer wants to introduce a recycled/remarked IC as a legitimate new product, then he will need to create a valid genesis transaction for the IC. However, such a transaction can only be created by the original IP owner registered with the consortium and can be easily monitored. The false genesis transaction would immediately be flagged and would incriminate the counterfeiter. In addition, the prevention of double spending [62] ensures that the counterfeiter cannot introduce multiple forged ICs by pretending to be a legitimate buyer and then, attempting a future sale.

If a registered manufacturer wants to introduce a recycled/remarked IC as a legitimate product, then it has to include the device identifier information in the transaction. But an already registered IC in the blockchain with the same device identifier would prevent the recycled IC to re-enter into the supply chain as a new one.

\subsection{Overproduced ICs}

In overproduction, untrusted foundry, assembly, or test site that have access to a designer's IP, may overproduce the original IP design outside the contract and the IP's owner knowledge. They can then sell the overproduced ICs in the open market as the original owner. Our proposed protocol defeats this malicious approach by having requirement that the registering manufacturer has to sign the transaction, registerDevice() using its private key. As the counterfeit manufacturer cannot get the original manufacturer's key, it cannot register the overproduced ICs as the original manufacturer.

If the counterfeiter decides to use his own brand (and own private key), then there is still a need to protect the original designer's Intellectual Property. In this case, IC metering [4], obfuscation/ locking [68], and/or Secure Split Test (SST)-based [63] methods can complement our proposed approach. These approaches use an activation key to uniquely activate each chip. Figure 6 shows how these approaches can complement our proposed scheme. After the manufacturer fabricates a chip, the designer sends the chip activation key via a secure link established by the designer and manufacturer's public key infrastructure (step 1 in Figure 6). After the chip gets the activation key, it is unlocked for testing and enrollment of PUF authentication data (step 2 in Figure 6). Finally, the original designer sends a transaction registerDevice() to the smart contract OC by signing the chip authentication data with its private key (step 3 in Figure 6). Recently, locking techniques have been targeted by SAT attacks [89]. These SAT attacks against locking techniques present a case for better solution.

\subsection{Cloned ICs and Tampered ICs}

The current owner cannot clone an IC with same ID and provenance information due to the presence of the embedded PUF whose data is recorded in the blockchain. Each PUF device has a unique 


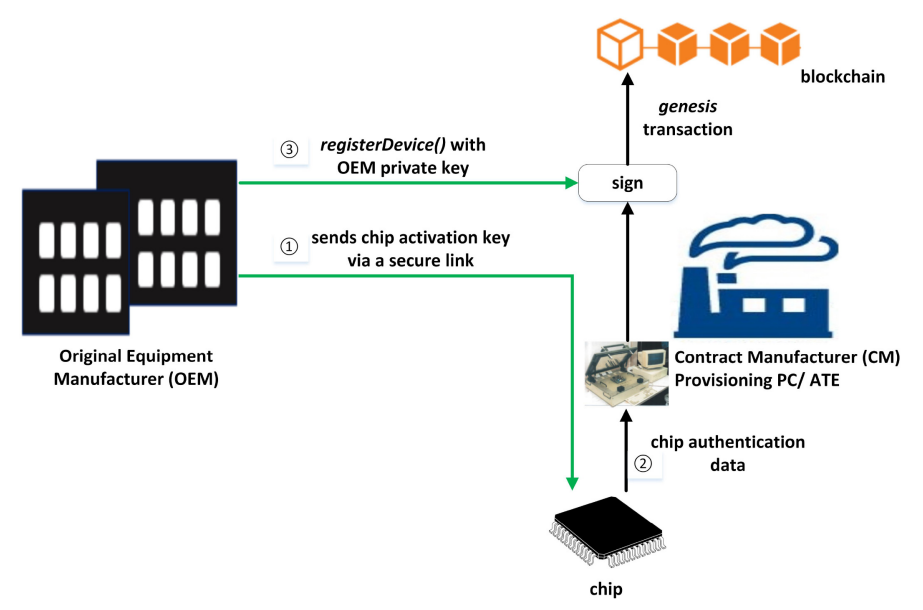

Fig. 6. Chip activation using active metering [68] before enrollment of authentication data in blockchain. Green arrow indicates secure link established by the designer and manufacturer's public key infrastructure. ATE: Automated Test Equipment.

and unpredictable way of mapping challenges to responses, even if it was manufactured with the same process as a similar device, and it is infeasible to clone a PUF with the same ChallengeResponse behavior as another given PUF. Sufficient PUF data is assumed to be available in the blockchain to assure the authenticity of the IC. Furthermore, our proposed method makes the tampering with the IC very costly due to the presence of the embedded PUF. Various PUF tampering techniques, such as, Focused Ion Beam (FIB) are costly and would have to be performed on a per chip basis to obtain multiple clones [68].

\section{ANALYSIS OF THE PROTOCOL}

In this section, we analyze the security, privacy, and reliability issues of the proposed blockchainbased IC traceability protocol, and we discuss the performance of our proposed protocol in terms of scalability and resource requirements.

\subsection{Security}

- Double spending by seller: Once the transaction is complete and added to the blockchain by a seller, the buyer becomes the only person authorized to update the blockchain for a particular IC. Hence, the seller cannot falsely attempt another sale.

- Seller's refusal to transfer IC: Following the previous point, the buyer can use the blockchain to take legal action against the seller if the seller refuses to transfer the IC to the buyer. The integrity and legitimacy of a supply chain participant is ensured during registration when it joins the system and gets its private/public keys (Section 4.2.1).

- De-centralized verification and authentication: In a traditional authentication process, a centralized Verification Authority (or Verifier) possesses a large set of CRPs for each IC, enrolled prior to sale. Authentication of a device in the field can only be performed by the Verifier. Our protocol provides the flexibility for any potential buyer to authenticate a chip.

- Modeling attack resistance: The buyer of an IC verifies CRPs on the actual physical device against the records in blockchain tracing back all the way to manufacturer. In this instance, a software model [71] of the PUF is irrelevant and cannot be used to mount an attack. Unless the chip is provisioned with an Artificial Neural Network (ANN), it cannot clone the PUF. 
Provisioning a chip with ANN, solely for the purpose of cloning increases die area and accrues additional cost. If an attacker chooses this route, then there will be a cost explosion, since software model of each chip has to be learned separately.

- Security against malicious foundries: If a third party/contract manufacturer (foundry) or assembly wants to behave badly, then our proposed approach gives no incentive to do that. For example, if a foundry creates a bad genesis transaction that is not authenticated by the on-chip PUF, it cannot sell that part. If the foundry creates a genesis transaction, which is authenticated by the PUF, but the chip is bad one, then the buyer knows precisely who is responsible. Besides, if the manufacturer provides incorrect PUF data, the IP owner cannot sell the chip and loses trust in the manufacturer. Moreover, if the manufacturer leaks the PUF data, still it is infeasible to build a hardware with a cloned PUF. Any effort by a malicious foundry to overproduce ICs is defeated by the locking scheme described in Section 7.2. Thus, the manufacturer has no incentive to become an attacker.

- Consortium blockchain security: The consortium members control the consensus process and write the consortium blockchain with transactional data. Since the customers cannot participate in the consensus process, they cannot compromise the security and privacy of consortium blockchain.

\subsection{Privacy}

- Transparency vs. privacy: The proposed protocol shows that an entity performs all the transactions using a single address. This may raise some privacy concerns for the customers. One possible approach to address the issue is to assign multiple addresses to each participant.

- Consortium blockchain vs. public blockchain: There are several advantages of a consortium blockchain over public blockchain. First, if the read permissions are restricted, consortium blockchain can provide a greater level of privacy [9]. Moreover, the consortium can, if desired, change the rules of the blockchain. Since the transactions need to be verified only by a select number of nodes, transactions are cheaper in terms of validation overheads. Any accidental fault can be quickly fixed, as the nodes are very well connected.

\subsection{Reliability}

- Authentication by Strong PUFs: The genesis block is assumed to have enough PUF data to disambiguate the IC from millions of other devices [64]. To increase the number of authentication events, the PUF can be re-mined during each ownership transfer event and the new data can be included in challenge and hashResponse fields of the transaction format. The hashing of PUF responses prevents their exposure in the blockchain and ensures only the physical owners of the IC can re-generate the hash. The remining process can also check the reliability of the PUF, which can be affected by aging over the product lifetime and update the blockchain accordingly.

- Authentication by Weak PUFs: To generate stable keys from the Weak PUF, various solutions have been proposed, such as, accelerated device aging [38, 39], built-in self-test [8], Helper Data Algorithm, also known as a Fuzzy Extractor [17], and so on. The helper data can be generated during enrollment phase and stored in a non-volatile memory present in the chip. It can be constructed in such a way that it can become known to an adversary without compromising the secrecy of the PUF response, i.e., it does not need to be kept secret $[20,69]$. During authentication stage, the secret key can be reconstructed combining the response from the PUF and helper data from the non-volatile memory. Alternatively, a non-volatile memory-based PUF can be used for authentication, which does not require any helper data [12]. If PUF is sufficiently reliable, then producing helper data may be omitted. 
Table 1. Operation Cost for a Chip in Supply Chain

\begin{tabular}{|c|c|c|c|c|}
\hline Operation & Gas limit & Gas price & Cost (in ETH) & Cost (in USD) \\
\hline registerDevice() & 121,478 & $10.89 \times 10^{-9}$ & 0.0013229 & 0.11 \\
\hline transferOwnership() & 30,365 & $10.89 \times 10^{-9}$ & 0.00033067 & 0.03 \\
\hline
\end{tabular}

\subsection{Performance}

- Scalability: Multiple ICs can be sold in a single transaction between two parties by utilizing the multiple inputs option in the transaction format. For online/remote sales, the buyer/seller can designate a trusted agent/broker.

- Low power and low cost authentication: The PUF consumes dynamic power only during authentication process-when it generates the response. The power required during authentication is small. Also, the PUF is a lightweight root-of-trust, enabling easier integration.

- Simple, robust authentication: PUFs enable fast, secure authentication of the ICs due to their unclonability and light footprint. Furthermore, the PUF information can be updated in the blockchain during each transfer and provides a future buyer with more data points to verify IC authenticity.

- Flexible consensus mechanisms: Permissioned platforms have semi-trusted members where only known participating nodes that are part of a consortium, are verified and registered. These groups are expected to be small in number and therefore can employ alternative consensus mechanisms. Achieving consensus in a distributed system has known solutions in the research literature, e.g., Paxos [51], RAFT [60], and various Byzantine Fault Tolerance algorithms [11]. Permissioned blockchain platforms have largely adopted these consensus algorithms.

\section{PROTOCOL DEMONSTRATION AND DISCUSSION}

In this section, we present the implementation of our proposed smart contract, operational cost of the consortium blockchain, limitations, practical use of the protocol, and possible future works.

\subsection{Smart Contract Implementation}

The proposed protocol has been implemented in Solidity scripting language and evaluated in terms of its operational cost. The code has been made publicly accessible in Github [1]. In particular, the total cost has been estimated by measuring the total gas amount (execution fee for every operation made on Ethereum) for all of the functions involved in the process, that is, (i) registering device (registerDevice()), and (ii) transferring ownership (transferOwnership()), and then converting it into a real currency. As the amount of gas is fixed for each operation in Ethereum, e.g., an SHA3 calculation costs 20 gas, the total gas amount for executing a function is also fixed. In particular, we have used the Ethereum's test environment tool, testrpc [41], to measure the gas amount, since this tool has the ability of automatically counting the gas amount. With current value of $1 \mathrm{ETH}=83.15 \mathrm{USD}$ and $1 \mathrm{Gas}=10.89 \times 10^{-9} \mathrm{ETH}(10.89 \mathrm{Gwei})$, the operation cost for a chip in supply chain is calculated in Table 1 . Finally, the total cost of maintaining the identity of a chip in a supply chain with $\mathrm{N}$ entities is

$$
\text { total cost }=\text { cost of enrollment }+(N-1) \times \text { cost of ownership transfer. }
$$

Using the formula, the total cost of maintaining identity of a chip in the supply chain presented in Figure 1 with five entities (manufacturer, distributor, retailer, consumer 1, consumer 2) is 0.11 USD $+0.03 \times 4=0.23$ USD. 


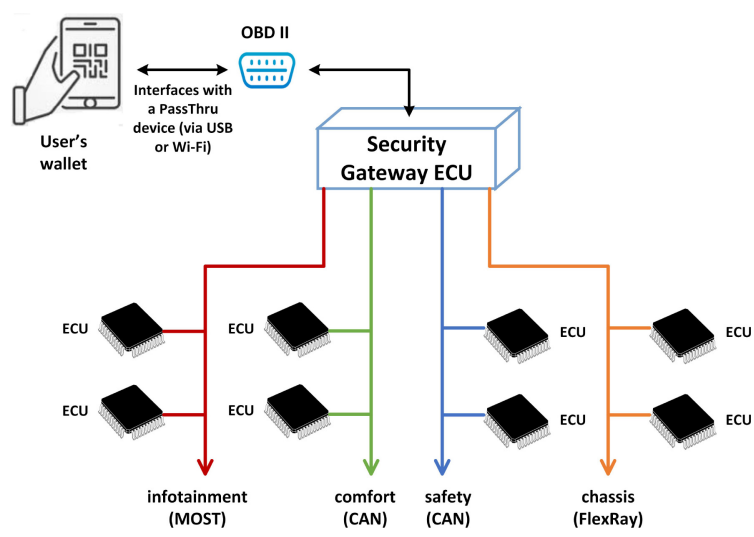

Fig. 7. Schematic of typical in-vehicle network architecture of a modern automobile. All inter-bus communication is done exclusively only over the gateway ECU. CAN-Controller Area Network, MOST-Media Oriented Systems Transport.

For IC authentication utilizing Strong PUFs, the number of PUF challenges required to distinguish 1 trillion ICs is calculated to be $\sim 1,024$ for a Hamming distance threshold of $10 \%$ [64]. For practical implementation, using, e.g., a 128-bit arbiter PUF [79], a set of 128 challenges can be taken and MD5-128 hash can be applied to the corresponding 128-bit responses [54]. This adds to total 2,176 bytes of data in the challenge, hashResponse fields of a transaction.

\subsection{Operational Cost of Consortium Blockchain}

Costs linked with the system activity such as the system set-up, its execution or maintenance are shared within the consortium according to a pre-established governance scheme. The consortium's incentive for building and maintaining blockchain infrastructure is to detect counterfeits in IC supply chain. Individual companies can leverage the same resources for various other blockchain applications. Additionally, for regular system maintenance, a consortium node is rewarded a small fee (e.g., some ETH) when it validates any transaction committed by a customer for ownership transfer. Selecting appropriate values for transfer reward will depend on the actual investment made by the consortium members for the implementation. However, such a topic is outside the scope of our current research, and thus it will not be considered further.

\subsection{Practicality of the Proposed Protocol}

Our proposed authentication, and the overall traceability protocol is highly suitable for a computing system where the IC is part of a system, such as System on Chips (SOCs), computer motherboard systems, automotive ECUs, and so on. These systems can be connected to the Internet and using our blockchain protocol allows all the ICs of the system to be authenticated simultaneously.

Figure 7 presents how the automotive security can be ensured during any ownership transfer using our IC traceability protocol via blockchain pegged to embedded Weak PUFs. Modern automobiles contain more than 70 Electronic Control Units (ECUs) networked together [73]. The overall safety of the vehicle relies on the authenticity of various ECUs. Security of a car can be compromised by connecting counterfeit ECU in vehicle communication network. A counterfeit ECU from any malicious party can threaten the functioning of steering, brakes, airbags, windows, headlights, and so on [87]. This gives rise to safety concern of people in and around the car. To circumvent this, we propose our traceability protocol, by which a buyer can authenticate the provenance of all ECUs during any ownership transfer. 
In the proposed protocol, a centralized gateway ECU connects all existing bus systems with each other. During device enrollment, all accredited OEMs (Original Equipment Manufacturer) of the respective vehicle enroll the ECUs by issuing registerDevice(). The vehicle manufacturer enlists device IDs of all ECUs in the gateway security ECU. At any stage of supply chain, a potential buyer can verify the ownership and the provenance of the ECUs by invoking checkOwnership() with the list of device IDs obtained form the gateway security ECU. He can authenticate all the ECUs connected in the vehicle network by invoking authenticateDevice().

For authentication, the blockchain sends public keys of all ECUs to the buyer's wallet. The buyer's wallet sends a challenge message to the gateway security ECU via a PassThru device [73]. The gateway ECU collects the signatures from all ECUs, including its own and sends back to buyer's wallet. The buyer's wallet can verify the digital signature for the authenticity of the device.

\subsection{Limitations}

Cloning PUFs: Recent work has shown that PUFs can be tampered by Focused Ion Beam (FIB) to create clones [30]. However, FIB-based tampering is costly and would have to be performed on a per chip basis to obtain multiple clones. Also, The authors of Reference [68] conjectured that FIB attack might not be possible for ICs with 32nm or smaller feature nodes.

Authenticity of Analog and Mixed-signal Circuits: The number of counterfeit analog ICs has been increasing at an alarming rate [77]. Our proposed solution is for ICs with embedded PUFs and is agnostic to the type of the device, whether analog, digital or mixed-signal. The solution is easily extended to analog and mixed-signal circuits (with analog PUFS [19], or package IDs [15, 49, 55]) but is outside the scope of the current article. Since the blockchain is pegged to PUF for authentication, legacy devices without any authentication mechanism are not supported by this solution.

\subsection{Future Directions}

Transparency vs. Privacy: An open research question regarding our proposed protocol is how both transparency requirement for supply chain tracking and anonymity requirement for maintaining user's privacy can be satisfied. This is of particular importance to IP owners selling millions of devices as it can be infeasible to create millions of public-private key pairs. In future works, we plan to investigate possible solutions to address this issue.

\section{CONCLUSION}

In this article, we outline a novel methodology to establish IC traceability using blockchain technology and PUFs. The blockchain allows legitimate parties to track an IC over its entire lifetime. Embedded PUFs are used to provide a simple, secure, and robust authentication process at every point-of-sale. The entire process of authenticating and ownership transfer has been automated by using smart contract. Our approach does not impose onerous restrictions on the participants in the blockchain; instead, the underlying technology guarantees the integrity of the system even in the face of dishonesty or idleness. This provides a technological solution to a supply chain problem.

\section{REFERENCES}

[1] Md Nazmul Islam. 2018. Blockchain for IC traceability. Retrieved from https://github.com/nazmulislam025/ICTraceability-Blockchain.

[2] Shannon Kempe. 2015. Data quality: It's a supply chain issue. Retrieved from http://www.dataversity.net/dataquality-its-a-supply-chain-issue/.

[3] Katelyn Rogers. 2013. Who is hiding behind the barcode? Retrieved from https://product.okfn.org/2013/10/16/ who-is-hiding-behind-the-barcode/.

[4] Yousra Alkabani and Farinaz Koushanfar. 2007. Active hardware metering for intellectual property protection and security. In Proceedings of the USENIX Security Symposium. 291-306. 
[5] Anastacia B. Alvarez, Wenfeng Zhao, and Massimo Alioto. 2016. Static physically unclonable functions for secure chip identification with $1.9-5.8 \%$ native bit instability at $0.6-1 \mathrm{~V}$ and $15 \mathrm{fJ} / \mathrm{bit}$ in $65 \mathrm{~nm}$. IEEE $\mathrm{f}$. Solid-State Circ. 51,3 (2016), 763-775.

[6] Daniel Augot, Hervé Chabanne, Olivier Clémot, and William George. 2017. Transforming face-to-face identity proofing into anonymous digital identity using the Bitcoin blockchain. arXiv preprint arXiv:1710.02951 (2017).

[7] Adam Back, Matt Corallo, Luke Dashjr, Mark Friedenbach, Gregory Maxwell, Andrew Miller, Andrew Poelstra, Jorge Timón, and Pieter Wuille. 2014. Enabling blockchain innovations with pegged sidechains. Retrieved from http://www. opensciencereview.com/papers/123/enablingblockchain-innovations-with-pegged-sidechains.

[8] Mudit Bhargava and Ken Mai. 2014. An efficient reliable PUF-based cryptographic key generator in 65nm CMOS. In Proceedings of the Conference on Design, Automation and Test in Europe. European Design and Automation Association, 70.

[9] Vitalik Buterin. 2015. On public and private blockchains. Retrieved from https://blog.ethereum.org/2015/08/07/ on-public-and-private-blockchains/.

[10] Vitalik Buterin et al. 2014. A next-generation smart contract and decentralized application platform. White Paper (2014).

[11] Miguel Castro, Barbara Liskov, et al. 1999. Practical Byzantine fault tolerance. In Proceedings of the Conference on Operating Systems Design and Implementation (OSDI'99), Vol. 99. 173-186.

[12] Wenjie Che, Jim Plusquellic, and Swarup Bhunia. 2014. A non-volatile memory-based physically unclonable function without helper data. In Proceedings of the IEEE/ACM International Conference on Computer-Aided Design. IEEE Press, $148-153$.

[13] Wenjie Che, Fareena Saqib, and Jim Plusquellic. 2015. PUF-based authentication. In Proceedings of the IEEE/ACM International Conference on Computer-Aided Design (ICCAD’15). IEEE, 337-344.

[14] Quynh H. Dang. 2012. Secure hash standard (SHS). Federal Inf. Process. Stds. (NIST FIPS)-180-4.

[15] DARPA. 2014. Supply chain hardware basic concepts and taxonomy of dependable and secure computing. Microsystems Technology Office/MTO Broad Agency Announcement.

[16] Kevin Delmolino, Mitchell Arnett, Ahmed Kosba, Andrew Miller, and Elaine Shi. 2016. Step by step towards creating a safe smart contract: Lessons and insights from a cryptocurrency lab. In Proceedings of the International Conference on Financial Cryptography and Data Security. Springer, 79-94.

[17] Jeroen Delvaux, Dawu Gu, Dries Schellekens, and Ingrid Verbauwhede. 2015. Helper data algorithms for PUF-based key generation: Overview and analysis. IEEE Trans. Comput.-Aided Design Integr. Circ. Syst. 34, 6 (2015), 889-902.

[18] Srinivas Devadas, Edward Suh, Sid Paral, Richard Sowell, Tom Ziola, and Vivek Khandelwal. 2008. Design and implementation of PUF-based "Unclonable" RFID ICs for anti-counterfeiting and security applications. In Proceedings of the IEEE International Conference on Radio Frequency ID (RFID'08). IEEE, 58-64.

[19] Sabyasachi Deyati, Abhijit Chatterjee, and Barry John Muldrey. 2017. Analog Push Pull Amplifier-Based Physically Unclonable Function for Hardware Security. U.S. Patent App. 15/336,895.

[20] Yevgeniy Dodis, Rafail Ostrovsky, Leonid Reyzin, and Adam Smith. 2008. Fuzzy extractors: How to generate strong keys from biometrics and other noisy data. SIAM J. Comput. 38, 1 (2008), 97-139.

[21] Ali Dorri, Salil S. Kanhere, and Raja Jurdak. 2017. Towards an optimized blockchain for IoT. In Proceedings of the 2nd International Conference on Internet-of-Things Design and Implementation. ACM, 173-178.

[22] Kaoutar Elkhiyaoui, Erik-Oliver Blass, and Refik Molva. 2012. CHECKER: On-site checking in RFID-based supply chains. In Proceedings of the 5th ACM Conference on Security and Privacy in Wireless and Mobile Networks. ACM, 173-184.

[23] Thomas Fay and Dominick Paniscotti. 2016. Systems and Methods of Blockchain Transaction Recordation. U.S. Patent App. 15/086,801.

[24] Danilo Gligoroski. 2010. Length extension attack on narrow-pipe SHA-3 candidates. In Proceedings of the International Conference on ICT Innovations. Springer, 5-10.

[25] Jorge Guajardo, Sandeep S. Kumar, Geert-Jan Schrijen, and Pim Tuyls. 2007. Physical unclonable functions and publickey crypto for FPGA IP protection. In Proceedings of the International Conference on Field Programmable Logic and Applications (FPL'07). IEEE, 189-195.

[26] Guardtime and IntrinsicID. [n.d.]. Internet of things authentication: A Blockchain solution using SRAM physical unclonable functions. Retrieved from https:/www.intrinsic-id.com/wp-content/uploads/2017/05/gt_KSI-PUF-web1611.pdf.

[27] Ujjwal Guin, Ke Huang, Daniel DiMase, John M. Carulli, Mohammad Tehranipoor, and Yiorgos Makris. 2014. Counterfeit integrated circuits: A rising threat in the global semiconductor supply chain. Proc. IEEE 102, 8 (2014), 1207-1228.

[28] Héléna Handschuh and Pim Theo Tuyls. 2011. Device and Method for Obtaining a Cryptographic Key. U.S. Patent App. 13/574,311. 
[29] Harvard Business Review. [n.d.]. The transparent supply chain. Retrieved from https://hbr.org/2010/10/thetransparent-supply-chain.

[30] Clemens Helfmeier, Christian Boit, Dmitry Nedospasov, and Jean-Pierre Seifert. 2013. Cloning physically unclonable functions. In Proceedings of the IEEE International Symposium on Hardware-Oriented Security and Trust (HOST'13). IEEE, 1-6.

[31] Petri Helo and Bulcsu Szekely. 2005. Logistics information systems: An analysis of software solutions for supply chain co-ordination. Industr. Manage. Data Syst. 105, 1 (2005), 5-18.

[32] Daniel E. Holcomb, Wayne P. Burleson, and Kevin Fu. 2009. Power-up SRAM state as an identifying fingerprint and source of true random numbers. IEEE Trans. Comput. 58, 9 (2009), 1198-1210.

[33] Seyoung Huh, Sangrae Cho, and Soohyung Kim. 2017. Managing IoT devices using blockchain platform. In Proceedings of the 19th International Conference on Advanced Communication Technology (ICACT'17). IEEE, 464467.

[34] Md Nazmul Islam and Sandip Kundu. 2016. Modeling residual lifetime of an ic considering spatial and inter-temporal temperature variations. In Proceedings of the IEEE 25th Asian Test Symposium (ATS'16). IEEE, 240-245.

[35] Md Nazmul Islam and Sandip Kundu. 2017. An analytical model for predicting the residual life of an IC and design of residual-life meter. In Proceedings of the IEEE 35th VLSI Test Symposium (VTS'17). IEEE, 1-6.

[36] Md Nazmul Islam and Sandip Kundu. 2018. Preserving IoT privacy in sharing economy via smart contract. In Proceeedings of the IEEE/ACM 3rd International Conference on Internet-of-Things Design and Implementation (IoTDI'18). IEEE, 296-297.

[37] Md Nazmul Islam, Vinay C. Patii, and Sandip Kundu. 2018. On IC traceability via blockchain. In Proceedings of the International Symposium on VLSI Design, Automation and Test (VLSI-DAT'18). IEEE, 1-4.

[38] Md Nazmul Islam, Vinay C. Patil, and Sandip Kundu. 2017. A guide to graceful aging: How not to overindulge in post-silicon burn-in for enhancing reliability of weak PUF. In Proceedings of the IEEE International Symposium on Circuits and Systems (ISCAS'17). IEEE, 1-4.

[39] Md Nazmul Islam, Vinay C. Patil, and Sandip Kundu. 2018. On enhancing reliability of weak PUFs via intelligent post-silicon accelerated aging. IEEE Trans. Circ. Syst. I: Reg. Papers 65, 3 (2018).

[40] ISO. 1995. EN ISO 8492.1995. European Committee for Standardization, Point 3.16.

[41] Kedar Iyer and Chris Dannen. 2018. The ethereum development environment. In Building Games with Ethereum Smart Contracts. Springer, 19-36.

[42] Monique H. Jansen-Vullers, Christian A. van Dorp, and Adrie J. M. Beulens. 2003. Managing traceability information in manufacture. Int. f. Info. Manage. 23, 5 (2003), 395-413.

[43] Chenglu Jin and Marten van Dijk. 2019. Secure and efficient initialization and authentication protocols for SHIELD. IEEE Trans. Depend. Secure Comput. 16, 1 (2019).

[44] Don Johnson, Alfred Menezes, and Scott Vanstone. 2001. The elliptic curve digital signature algorithm (ECDSA). Int. f. Info. Secur. 1, 1 (2001), 36-63.

[45] I. Kar. 2016. Estonian Citizens Will Soon Have the World's Most Hack-Proof Health-Care Records. Retrieved from https://qz.com/628889/this-eastern-european-country-is-moving-its-health-records-to-the-blockchain/.

[46] J. Kelly and A. Williams. 2016. Forty Big Banks Test Blockchain-Based Bond Trading System. Retrieved from https://www.reuters.com/article/banking-blockchain-bonds/forty-big-banks-test-blockchain-based-bond-tradingsystem-idUSL8N16A30H.

[47] L. W. Kessler and T. Sharpe. 2010. Faked parts detection. Retrieved from http://www.circuitsassembly.com/cms/ component/content/article/159/9937-smt.

[48] Robin Koh, Edmund W. Schuster, Indy Chackrabarti, and Attilio Bellman. 2003. Securing the pharmaceutical supply chain. White Paper, Auto-ID Labs, Massachusetts Institute of Technology (2003), 1-19.

[49] Cyrill Kuemin, Lea Nowack, Luisa Bozano, Nicholas D. Spencer, and Heiko Wolf. 2012. Oriented assembly of gold nanorods on the single-particle level. Adv. Funct. Mater. 22, 4 (2012), 702-708.

[50] S. Lacey. 2016. The energy blockchain: How bitcoin could be a catalyst for the distributed grid. Retrieved from https://www.greentechmedia.com/articles/read/the-energy-blockchain-could-bitcoin-be-a-catalyst-for-thedistributed-grid.

[51] Leslie Lamport. 2005. Generalized consensus and Paxos.

[52] Jae W. Lee, Daihyun Lim, Blaise Gassend, G. Edward Suh, Marten Van Dijk, and Srinivas Devadas. 2004. A technique to build a secret key in integrated circuits for identification and authentication applications. In Proceedings of the Symposium on VLSI Circuits. IEEE, 176-179.

[53] Yuan Liu, Zheng Zhao, Guibing Guo, Xingwei Wang, Zhenhua Tan, and Shuang Wang. 2017. An identity management system based on blockchain. Proceedings of the Conference on Privacy, Security and Trust (PST'17).

[54] Cheryl Madson and Rob Glenn. 1998. The use of HMAC-MD5-96 within ESP and AH (No. RFC 2403). 
[55] Mitchell Miller, Janice Meraglia, and James Hayward. 2012. Traceability in the Age of Globalization: A Proposal for a Marking Protocol to Assure Authenticity of Electronic Parts. Technical Report. SAE Technical Paper.

[56] Alex Mizrahi. 2015. A Blockchain-based Property Ownership Recording System.

[57] Satoshi Nakamoto. 2008. Bitcoin: A Peer-to-peer Electronic Cash System.

[58] C. Ninlawan, P. Seksan, K. Tossapol, and W. Pilada. 2010. The implementation of green supply chain management practices in electronics industry. In Proceedings of the International Multiconference of Engineers and Computer Scientists, Vol. 3. 17-19.

[59] U.S. Senate Committee on Armed Services. May 2012. Inquiry into counterfeit electronic parts in the Department of Defence supply chain. Retrieved from https:/www.armed-services.senate.gov/imo/media/doc/ Counterfeit-Electronic-Parts.pdf.

[60] Diego Ongaro and John K. Ousterhout. 2014. In search of an understandable consensus algorithm. In Proceedings of the USENIX Annual Technical Conference. 305-319.

[61] Michael Pecht and Sanjay Tiku. 2006. Bogus: Electronic manufacturing and consumers confront a rising tide of counterfeit electronics. IEEE Spectrum 43, 5 (2006), 37-46.

[62] David Pointcheval and Jacques Stern. 2000. Security arguments for digital signatures and blind signatures. 7 . Cryptol. 13, 3 (2000), 361-396.

[63] Md Tauhidur Rahman, Domenic Forte, Quihang Shi, Gustavo K. Contreras, and Mohammad Tehranipoor. 2014. CSST: Preventing distribution of unlicensed and rejected ICs by untrusted foundry and assembly. In Proceedings of the IEEE International Symposium on Defect and Fault Tolerance in VLSI and Nanotechnology Systems (DFT'14). IEEE, 46-51.

[64] P. Ramesh, V. C. Patil, and S. Kundu. 2017. Peer pressure on identity: On requirements for disambiguating PUFs in noisy environment. In Proceedings of the IEEE North Atlantic Test Workshop (NATW'17). 1-4.

[65] Damith Ranasinghe, Daniel Engels, Peter Cole, et al. 2004. Security and privacy: Modest proposals for low-cost RFID systems. In Proceedings of the Auto-ID Labs Research Workshop.

[66] Pete Rizzo. July, 2015. Blockchain identity startup shocard raises 1.5 million. Retrieved from http://www.coindesk. com/blockchain-identity-startup-shocard-1-5-million/.

[67] Norm Robson, John Safran, Chandrasekharan Kothandaraman, Alberto Cestero, Xiang Chen, Raj Rajeevakumar, Alan Leslie, Dan Moy, Toshiaki Kirihata, and Subramanian Iyer. 2007. Electrically programmable fuse (efuse): From memory redundancy to autonomic chips. In Proceedings of the Custom Integrated Circuits Conference (CICC'07). IEEE, 799-804.

[68] Jarrod A. Roy, Farinaz Koushanfar, and Igor L. Markov. 2010. Ending piracy of integrated circuits. Computer 43, 10 (2010), 30-38.

[69] Ulrich Rührmair and Daniel E. Holcomb. 2014. PUFs at a glance. In Proceedings of the Conference on Design, Automation, and Test in Europe. European Design and Automation Association, 347.

[70] Ulrich Rührmair, Frank Sehnke, Jan Sölter, Gideon Dror, Srinivas Devadas, and Jürgen Schmidhuber. 2010. Modeling attacks on physical unclonable functions. In Proceedings of the 17th ACM Conference on Computer and Communications Security (CCS'10). ACM, New York, NY, 237-249.

[71] Ulrich Rührmair, Jan Sölter, Frank Sehnke, Xiaolin Xu, Ahmed Mahmoud, Vera Stoyanova, Gideon Dror, Jürgen Schmidhuber, Wayne Burleson, and Srinivas Devadas. 2013. PUF modeling attacks on simulated and silicon data. IEEE Trans. Info. Forens. Secur. 8, 11 (2013), 1876-1891.

[72] Jon Russell. 2017. IBM is using the blockchain to speed up and simplify cross-border payments. Retrieved from https://techcrunch.com/2017/10/16/ibm-cross-border-payments-blockchain/.

[73] Florian Sagstetter, Martin Lukasiewycz, Sebastian Steinhorst, Marko Wolf, Alexandre Bouard, William R. Harris, Somesh Jha, Thomas Peyrin, Axel Poschmann, and Samarjit Chakraborty. 2013. Security challenges in automotive hardware/software architecture design. In Proceedings of the Conference on Design, Automation, and Test in Europe. EDA Consortium, 458-463.

[74] Jie Shi, Su Mon Kywe, and Yingjiu Li. 2014. Batch clone detection in RFID-enabled supply chain. In Proceedings of the IEEE International Conference on Radio Frequency ID (RFID'14). IEEE, 118-125.

[75] Ken Shirriiff. 2014. Bitcoins the hard way: Using the raw Bitcoin protocol. Retrieved from http://www.righto.com/ 2014/02/bitcoins-hard-way-using-raw-bitcoin.html.

[76] Joseph P. Skudlarek, Tom Katsioulas, and Michael Chen. 2016. A platform solution for secure supply-chain and chip life-cycle management. Computer 49, 8 (2016), 28-34.

[77] Solid State Technology. [n.d.]. Top 5 counterfeited semiconductors: Analog ICs top the list. Retrieved from https:// electroiq.com/2012/04/top-5-counterfeited-semiconductors-analog-ics-top-the-list/.

[78] Thorsten Staake, Frédéric Thiesse, and Elgar Fleisch. 2005. Extending the EPC network: The potential of RFID in anti-counterfeiting. In Proceedings of the ACM Symposium on Applied Computing. ACM, 1607-1612.

[79] G. Edward Suh and Srinivas Devadas. 2007. Physical unclonable functions for device authentication and secret key generation. In Proceedings of the 44th Annual Design Automation Conference. ACM, 9-14. 
[80] Melanie Swan. 2015. Blockchain: Blueprint for a New Economy. O’Reilly Media, Inc.

[81] Nick Szabo. 1997. Formalizing and securing relationships on public networks. First Monday 2, 9 (1997).

[82] Don Tapscott and Alex Tapscott. 2016. Blockchain Revolution: How the Technology Behind Bitcoin is Changing Money, Business, and the World. Penguin.

[83] Pim Tuyls and Lejla Batina. 2006. RFID-tags for anti-counterfeiting. In Cryptographers' Track at the RSA Conference. Springer, 115-131.

[84] Pim Tuyls and Boris Škorić. 2007. Strong authentication with physical unclonable functions. In Security, Privacy, and Trust in Modern Data Management. Springer, 133-148.

[85] A. Vijayakumar, V. C. Patil, C. B. Prado, and S. Kundu. 2016. Machine learning resistant strong PUF: Possible or a pipe dream? In Proceedings of the IEEE International Symposium on Hardware Oriented Security and Trust (HOST'16). $19-24$.

[86] MGCSA Walport. 2016. Distributed ledger technology: Beyond blockchain. UK Government Office for Science (2016).

[87] Armin Wasicek. 2014. Protection of intellectual property rights in automotive control units. SAE Int. F. Passenger Cars-Electron. Electric. Syst. 7, 2014-01-0338 (2014), 201-212.

[88] Roger Wattenhofer. 2016. The Science of the Blockchain. CreateSpace Independent Publishing Platform.

[89] Yang Xie and Ankur Srivastava. 2017. Delay locking: Security enhancement of logic locking against ic counterfeiting and overproduction. In Proceedings of the 54th Annual Design Automation Conference. ACM, 9.

[90] Kun Yang, Domenic Forte, and Mark Tehranipoor. 2015. An RFID-based technology for electronic component and system Counterfeit detection and traceability. In Proceedings of the IEEE International Symposium on Technologies for Homeland Security (HST'15). IEEE, 1-6.

[91] Kun Yang, Domenic Forte, and Mark Tehranipoor. 2018. ReSC: An RFID-enabled solution for defending IoT supply chain. ACM Trans. Design Auto. Electron. Syst. 23, 3 (2018), 29.

[92] Meng-Day Mandel Yu and Srinivas Devadas. 2017. Pervasive, dynamic authentication of physical items. Commun. ACM 60, 4 (2017), 32-39.

Received September 2018; revised February 2019; accepted February 2019 